\title{
CARACTERIZAÇÃO DA PRODUÇÃO DE SERAPILHEIRA E DA CHUVA DE SEMENTES EM UMA RESERVA DE FLORESTA ESTACIONAL SEMIDECIDUAL, PARANÁ
}

\author{
CHARACTERIZATION OF THE LITTERFALL PRODUCTION AND SEED RAIN IN A \\ RESERVE OF SEASONAL SEMIDECIDUOUS FOREST, PARANÁ STATE
}

\author{
Maria Angélica Gonçalves Toscan ${ }^{1}$ Ana Tereza Bittencourt Guimarães ${ }^{2}$ \\ Lívia Godinho Temponi²
}

\begin{abstract}
RESUMO
O objetivo do estudo foi analisar a produção de serapilheira e a chuva de sementes em um fragmento de Floresta Estacional Semidecidual do oeste do Paraná com 242 ha, que é conhecido como Reserva Particular de Patrimônio Natural Fazenda Santa Maria. O material foi coletado mensalmente de junho/2011 a maio/2012, em nove parcelas de 20 x $20 \mathrm{~m}$, com o uso quatro coletores de $0,5 \times 0,5 \mathrm{~m}$ em cada parcela. A serapilheira foi triada e pesada, e a chuva de sementes foi avaliada quanto à riqueza, forma de vida, categoria sucessional e síndrome de dispersão das espécies encontradas. A produção anual de serapilheira foi de $11.886 \mathrm{~kg} \mathrm{ha}^{-1}$, sendo agosto e setembro os meses de maiores produções. A fração foliar foi a mais representativa com $58,52 \%$ do total. Na chuva de sementes foram coletadas 18.300 sementes, distribuídas em 79 morfoespécies, das quais 51 foram identificadas em nível de espécie, oito em nível de gênero e seis em nível de família. Mikania sp., Cecropia pachystachya, Pisonia aculeata, Gouania ulmifolia e Dendropanax cuneatus foram as espécies com maiores densidades relativas. Os meses com maior abundância de sementes foram setembro (19\%), outubro (20\%), novembro (27\%) e março (15\%). A forma de vida predominante foi arbórea com $76,27 \%$ das espécies, seguida de lianas com 20,34\% e herbáceas com apenas 3,39\%. Entre as espécies arbóreas, 37\% foram representadas pela categoria sucessional de pioneiras, enquanto as categorias secundárias iniciais e tardias representaram $22 \%$ cada e as climácicas $20 \%$. A zoocoria predominou entre as síndromes de dispersão $(52,54 \%)$, enquanto a anemocoria e a autocoria ocorreram em 38,98 e 8,47\%, respectivamente. Os resultados foram semelhantes aos encontrados em outros trabalhos realizados em Florestas Estacionais Semideciduais tardias. Assim, por meio deste estudo sobre deposição da serapilheira e chuva de sementes, o fragmento florestal pode ser considerado uma área de floresta tardia e com elevado potencial de regeneração.
\end{abstract}

Palavras-chave: matéria orgânica, dispersão de sementes, zoocoria.

\begin{abstract}
This study aimed to analyze litterfall production and seed rain of a fragment of seasonal semideciduous forest from western of Parana state with 242 ha, which is known as Santa Maria

1 Bióloga, MSc., Doutoranda do Programa de Pós-Graduação em Ciências Biológicas, Universidade Estadual de Londrina, Rod. Celso Garcia Cid, PR 445, Km 380, Caixa Postal 10011, CEP 86057-970, Londrina (PR), Brasil. maria_angelica_gt@hotmail.com

2 Bióloga, Dra., Professora do Programa de Pós-Graduação em Conservação e Manejo de Recursos Naturais, Universidade Estadual do Oeste do Paraná, Rua Universitária 2069, Jardim Universitário, CEP 85819-110, Cascavel (PR), Brazil. liviatemponi@yahoo.com.br / anatbguimaraes@gmail.com
\end{abstract}


Farm's Private Nature Reserve. The material was collected from June/2011 to May/2012, in nine plots of $20 \times 20 \mathrm{~m}$, using four traps of $0,5 \times 0,5 \mathrm{~m}$ in each plot. The litter was sorted and weighed, and the seed rain was_evaluated as the wealth, life form, successional category and dispersal syndrome of the species found. The annual litterfall production was $11.886 \mathrm{~kg} \mathrm{ha}^{-1}$, being August and September the months of the largest production. The leaf fraction was the most representative with $58,52 \%$ of the total. In the seed rain were collected 18.300 seeds,_distributed in 79 morphospecies, which 51 were identified to species level, eight at the genus level and six at the family level. Mikania sp., Cecropia pachystachya, Pisonia aculeata, Gouania ulmifolia and Dendropanax cuneatus, were the species with the highest relative densities. The months with the highest abundance of seeds were September (19\%), October (20\%), November (27\%) and March (15\%). The life form predominant was tree with $76,27 \%$ of the species,_followed by climbers with 20,34\% and herbaceous with 3,39\% only. Among the tree species, $37 \%$ were represented by successional category of pioneer, while the early and late secondary categories accounted for $22 \%$ each and the climax 20\%. The zoochory predominated among dispersal syndromes (52,54\%), while anemochory and autocory occurred in $38,98 \%$ and $8,47 \%$, respectively. The results were similar to those found in other works performed in late seasonal semideciduous forest. Furthermore, through this study about litterfall production and seed rain, the forest fragment can be considered an area of mature forest and with high regeneration potential.

Keywords: organic matter, seed dispersal, zoochory.

\section{INTRODUÇÃO}

O processo de fragmentação causa redução e isolamento de habitat, levando a alterações na composição e distribuição das espécies, modificações na polinização, na dispersão das sementes e nas interações das espécies, o que prejudica a manutenção e dinâmica das mesmas, podendo levá-las à extinção (SCARIOT et al., 2005). Os efeitos da fragmentação estão diretamente relacionados com a matriz em que o fragmento está inserido, com o tamanho e como se distanciam uns dos outros (PRIMACK; RODRIGUES, 2001; MARTINS et al., 2012).

As Reservas Particulares de Patrimônio Natural (RPPN) são Unidades de Conservação (UC) localizadas em propriedades particulares e dependem que o proprietário se manifeste para o seu estabelecimento. Esses remanescentes de vegetação nativa são as UC mais abundantes do Brasil e apresentam como principal objetivo o de proteção de importantes fragmentos vegetais que acabam sendo pequenos para serem protegidos nas categorias federais ou estaduais, mas representam habitat importantes para a conservação da diversidade biológica (RYLANDS; BRANDON, 2005; FICAGNA, 2009).

Para que seja realizada a caracterização ecológica de áreas de conservação e restauração devem ser realizadas avaliações de indicadores ambientais (RODRIGUES; GANDOLFI, 1998). Entre esses indicadores destacam-se os estudos sobre fitossociologia, regeneração natural por indivíduos jovens, banco de sementes do solo, produção de serapilheira e chuva de semente (MARTINS, 2001). Com exceção dos estudos fitossociológicos, os demais podem ser utilizados como parâmetros funcionais responsáveis pelo restabelecimento das funções ecológicas do ecossistema em questão (ARAUJO, 2002).

A serapilheira depositada sobre o solo da floresta funciona como um sistema de entrada e saída de matéria orgânica. Ela recebe material principalmente da vegetação que, uma vez decomposto, fornece matéria orgânica para o solo e para as raízes (EWEL, 1976). Nos ambientes florestais, alguns padrões se repetem como a maior concentração de nutrientes nas folhas do que nos outros componentes da biomassa; tendência de produção mais elevada de serapilheira nos 
meses de menor fotoperíodo; o elevado grau de deciduidade das árvores e a sazonalidade da decomposição da serapilheira (PAGANO; DURIGAN, 2009).

A chuva de sementes representa chegada de diásporos, ou seja, sementes, frutos e esporos, de diferentes espécies a um ambiente, e o posterior estabelecimento desses diásporos direcionará o desenvolvimento sucessional e as mudanças na comunidade (HOWE; SMALLWOOD, 1982), definindo, portanto, o modelo para o futuro padrão de regeneração e recrutamento da população (LOISELLE et al., 1996). Portanto, estudos sobre a chuva de sementes fornecem informações sobre a abundância e riqueza das espécies que estão chegando a um local (GROMBONE-GUARATINI; RODRIGUES, 2002).

Dessa forma, o objetivo do estudo foi analisar a produção de serapilheira e a chuva de sementes em um fragmento de Floresta Estacional Semidecidual do oeste do Paraná.

\section{MATERIAL E MÉTODOS}

\section{Caracterização da área}

A área de estudo é conhecida como Reserva Particular do Patrimônio Natural (RPPN) Fazenda Santa Maria, um fragmento florestal de 242 ha, situada na cidade de Santa Terezinha de Itaipu, no oeste do Paraná. A RPPN Fazenda Santa Maria localiza-se no Corredor Ecológico de Santa Maria, que foi implementado em 2003 com o objetivo de ligar áreas entre o Parque Nacional do Iguaçu e a Faixa de Proteção do Reservatório de Itaipu por meio de fragmentos de diferentes tamanhos (Figura 1).

O clima da região é Subtropical Úmido Mesotérmico, Cfa segundo Köppen. A temperatura média anual é de $21^{\circ} \mathrm{C}$, os verões são quentes, apresentando médias superiores a $22^{\circ} \mathrm{C}$, e os invernos são frios, com médias inferiores a $18^{\circ} \mathrm{C}$. As precipitações são bem distribuídas ao longo do ano com precipitação anual de aproximadamente $1.800 \mathrm{~mm}$, com uma pequena redução no inverno, mas sem uma seca marcada (INSTITUTO AGRONÔMICO DO PARANÁ, 2012).

A formação vegetacional é de Floresta Estacional Semidecidual caracterizada por apresentar uma estação mais úmida, que é representada pela primavera e verão, e outra mais seca, quando pode ocorrer redução nas chuvas ou seca fisiológica causada pelas baixas temperaturas do outono e inverno. Tal fato leva ao aumento de quedas foliares, pois 20 a 50\% de suas espécies são decíduas, ou seja, espécies que perdem folhas nestas condições climáticas (IBGE, 2012).

\section{Coleta dos Dados}

Para a coleta do material utilizaram-se nove parcelas de $20 \times 20 \mathrm{~m}$ distribuídas lado a lado com $20 \mathrm{~m}$ de espaçamento entre elas. Para a análise da produção de serapilheira, cada parcela teve quatro coletores de $0,5 \times 0,5 \mathrm{~m}$ instalados de forma inteiramente casualizada, estes foram feitos com borda de madeira e tela de malha de $1 \mathrm{~mm}^{2}$.

A instalação dos coletores ocorreu em maio/2011 e o material neles depositado foi coletado mensalmente entre junho/2011 e maio/2012. A secagem de todo conteúdo coletado foi realizada em estufa de circulação forçada de ar a $70^{\circ} \mathrm{C}$, durante 48 horas. Posteriormente, esse passou por triagem nas seguintes frações: folhas, ramos, materiais reprodutivos (flores, frutos e sementes) e miscelânea (restos de animais, fezes, entre outros).

As frações foram pesadas em balança de precisão para a análise da deposição de serapilheira em relação às suas frações durante todo o período amostrado. Os valores de cada período de coleta foram transformados em $\mathrm{Kg} \mathrm{ha}^{-1}$ dia $^{-1}$ devido às variações nas datas de coleta em cada um dos meses. 


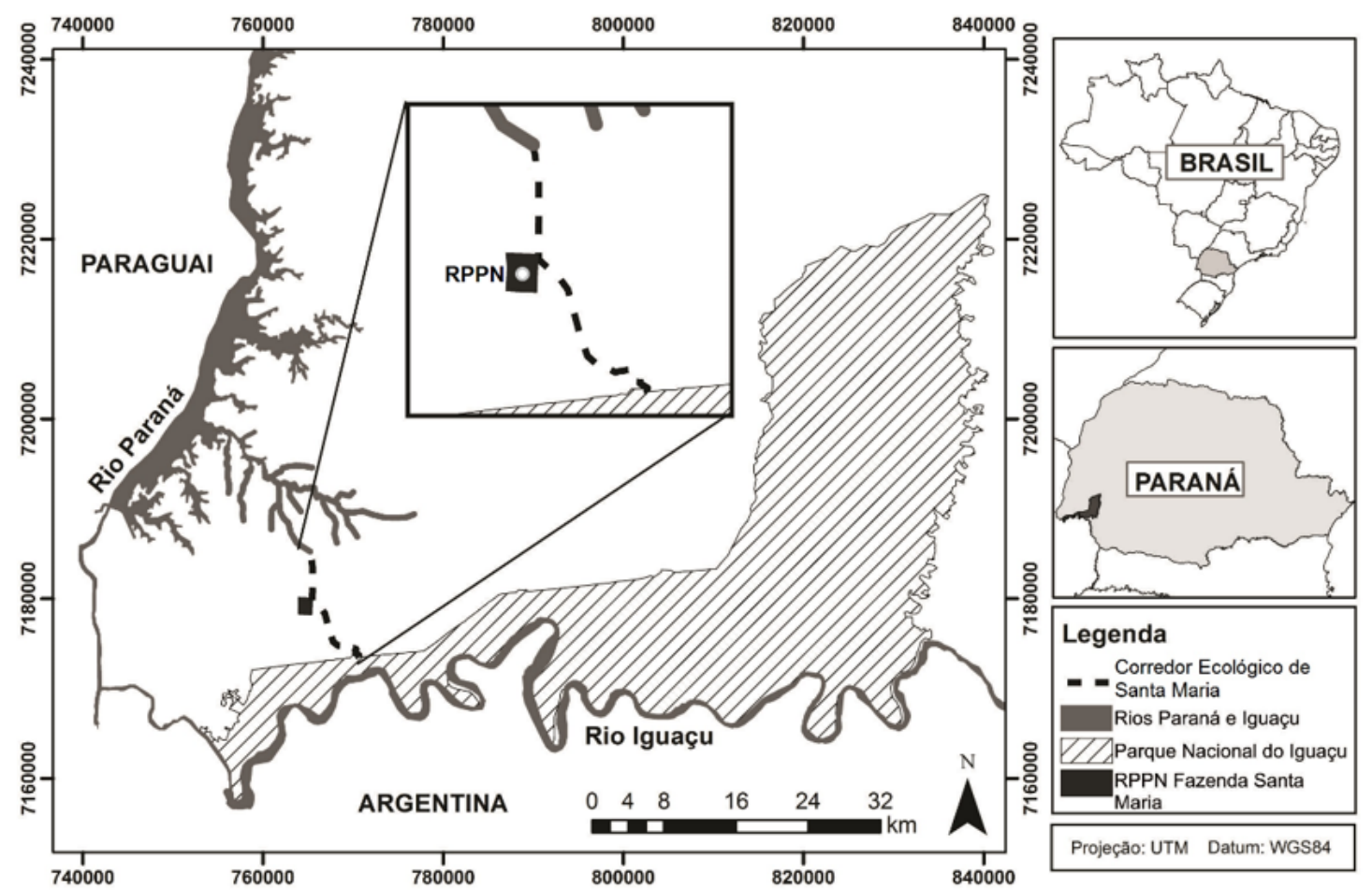

FIGURA 1: Mapa do Corredor Ecológico de Santa Maria - PR, mostrando a RPPN Fazenda Santa Maria no meio deste corredor.

FIGURE 1: Map of Santa Maria Ecologic Corridor, PR, showing the Santa Maria Farm's Private Nature Reserve in the middle of this corridor.

A caracterização da chuva de sementes ocorreu a partir da fração de materiais reprodutivos presente na serapilheira. A identificação foi realizada por meio de materiais bibliográficos, exsicatas, carpotecas e sementotecas de herbários e museus botânicos.

As sementes obtidas passaram por contagem, e para os frutos encontrados foi utilizada a quantidade média de sementes que o fruto da respectiva espécie apresentava. Para estimar esse valor foram utilizadas informações bibliográficas ou realizada a abertura dos frutos para a contagem de sementes. Os valores mensais foram ajustados para a média de sementes dia $^{-1}$.

As espécies identificadas na chuva de sementes foram classificadas em arbórea (incluindo as arbustivas), herbácea e liana, para a classificação na forma de vida. As sementes correspondentes às espécies arbóreas também tiveram sua classificação conforme a categoria sucessional, sendo pioneira, secundária inicial, secundária tardia e climácica (BUDOWSKI, 1965). E todas as espécies tiveram sua classificação referente à síndrome de dispersão de diásporos, que pode ser realizada por anemocoria, autocoria ou zoocoria (VAN DER PIJL, 1982).

\section{Análise dos Dados}

Para avaliar a diferença da produção de serapilheira mensal total e cada fração foi utilizada a análise do tipo Anova de medidas repetidas. Em caso de significância estatística, foi aplicado o teste de médias de Tukey a 0,05 de significância, para avaliar as diferenças entre as médias.

A densidade absoluta (DA) do total de sementes da chuva de sementes foi calculada, e para cada espécie amostrada foi calculada a densidade relativa (DR).

Para a comparação do número médio de sementes dia $^{-1}$ coletadas em cada mês foi realizado 
o Teste de Friedman (não paramétrico), na análise foram realizadas comparações pareadas de cada mês a fim de se detectar diferença entre os mesmos $(p<0,05)$.

A frequência relativa das sementes coletadas de cada forma de vida (arbóreas, lianas e herbáceas) e de cada síndrome e dispersão (zoocoria, anemocoria e autocoria) foram relacionadas graficamente com os meses de coleta, de forma a realizar uma análise temporal ao longo do período estudado. Isso foi realizado com o objetivo de detectar o assincronismo entre as espécies de arbóreas e lianas, que é característico de Florestas Estacionais Semideciduais tardias (ENGEL et al., 1998). Além de detectar a frequência de espécies zoocóricas durante o ano amostrado, que é indicativa de disponibilidade constante de frutos para a fauna associada, que como apontado por Howe e Smallwood (1982) esta deve ser a síndrome de dispersão predominante em florestas tropicais.

Para analisar a correlações entre valores mensais médios de serapilheira em $\mathrm{Kg} \mathrm{ha}^{-1} \mathrm{dia}^{-1} \mathrm{e}$ os de sementes dia ${ }^{-1}$ foi realizada uma Correlações de Spearman $\left(r_{s}\right)(Z A R, 1999)$.

\section{RESULTADOS E DISCUSSÃo}

A produção anual de serapilheira na RPPN Fazenda Santa Maria foi de $11.886 \mathrm{~kg} \mathrm{ha}^{-1}$, valor este semelhante aos obtidos em outros estudos em Florestas Estacionais Semideciduais. Pezzatto e Wisniewski (2006) obtiveram um total anual muito próximo do presente estudo, de $11.700 \mathrm{~kg} \mathrm{ha}^{-1}$ em uma Floresta Estacional Semidecidual secundária no oeste do Paraná; Machado et al. (2008) registraram $10.170 \mathrm{Kg} \mathrm{ha}^{-1}$, em uma Floresta Estacional Decidual no Rio de Janeiro e Vital et al. (2004) coletaram 10.646 Kg ha-1, em uma Floresta Estacional Semidecidual em Botucatu - SP.

A fração foliar foi a mais representativa, assim como apresentado de forma geral nos demais trabalhos comparados, correspondendo a 58,52\% (6.955,5 $\left.\mathrm{kg} \mathrm{ha}^{-1}\right)$ da fração total da serapilheira. A fração de ramos representou 28,16\% $\left(3.346,8 \mathrm{~kg} \mathrm{ha}^{-1}\right)$, os materiais reprodutivos 9,79\% (1.163,2 $\left.\mathrm{kg} \mathrm{ha}^{-1}\right)$ e a miscelânea 3,54\% (420,5 $\left.\mathrm{kg} \mathrm{ha}^{-1}\right)$. Segundo Pagano e Durigan (2009), as folhas representam o componente principal e determinante para a produção total da serapilheira.

Contudo, os meses com produções mais elevadas de serapilheira foram agosto (60,6 Kg ha ${ }^{-1}$ dia $\left.^{-1}\right)$ e setembro $\left(55,3 \mathrm{Kg} \mathrm{ha}^{-1} \mathrm{dia}^{-1}\right)$, apresentando médias significativamente superiores em relação a todos os demais meses analisados $(p<0,05)$ (Figura 2). Esses resultados corroboram com outros estudos em áreas de Floresta Estacional Semidecidual, em que as maiores produções ocorrem no fim do inverno. Martins e Rodrigues (1999) encontraram maiores produções entre agosto e novembro; Vital et al. (2004) evidenciaram a maior produção em setembro; e Pimenta et al. (2011) obtiveram picos de produções em setembro e outubro.

Em relação à fração foliar, os meses com maiores produções de folhas também foram agosto $\left(31,6 \mathrm{Kg} \mathrm{ha}^{-1}\right.$ dia $\left.^{-1}\right)$ e setembro $\left(33,3 \mathrm{Kg} \mathrm{ha}^{-1}\right.$ dia $\left.^{-1}\right)$ em relação aos demais meses $(\mathrm{p}<0,05)$ (Figura 2). Tal resultado demonstra que os meses do inverno (julho, agosto, setembro), de menores fotoperíodo e temperaturas, levam ao aumento da deciduidade, em 20 a 50\% das espécies presentes em Florestas Estacionais Semideciduais, fazendo com que ocorra um pico de produção de serapilheira por volta do mês de setembro marcando o fim dessa estação (IBGE, 2012; PAGANO; DURIGAN, 2009).

A fração de ramos também apresentou maiores produções em agosto (24,3 $\mathrm{Kg} \mathrm{ha}^{-1}$ dia $\left.^{-1}\right)$ e setembro $\left(16,9 \mathrm{Kg} \mathrm{ha}^{-1} \mathrm{dia}^{-1}\right)$ em relação aos demais meses $(\mathrm{p}<0,05)$. As frações de materiais reprodutivos e miscelânea não apresentaram diferenças significativas durante o período analisado $(\mathrm{p}>0,05)$ (Figura 2).

A chuva de sementes apresentou no total 18.300 sementes coletadas, estas pertenciam a 77 morfoespécies, sendo que 49 foram identificadas ao nível de espécie, oito ao nível de gênero e seis 
ao nível de família (Tabela 1).

Os valores de densidade absoluta de 2.033,29 sementes $\mathrm{m}^{-2}$ e de riqueza de espécies $(\mathrm{S}=79)$, registrados em um ano foram superiores aos obtidos em outros estudos com chuva de sementes realizados em fragmentos de Florestas Estacionais (PENHALBER; MANTOVANI, 1997; GROMBONE-GUARATINI; RODRIGUES, 2002; ARAUJO et al., 2004; CAMPOS et al., 2009) (Tabela 2). Os diferentes valores ocorreram devido às diferentes características, como diferenças florísticas nas distribuições regionais das espécies e diferentes estágios sucessionais dos fragmentos florestais comparados. Porém, trabalhos com chuva de sementes são escassos no Brasil e, em especial, no estado do Paraná, dificultando a comparação dos dados.

As espécies que apresentaram maiores valores de densidades relativas foram Mikania sp. (37,79\%), Cecropia pachystachya (14,86\%), Pisonia aculeata (12,40\%), Gouania ulmifolia (11,03\%) e Dendropanax cuneatus (6,54\%) (Tabela 1). Muitas espécies do gênero Mikania são representadas por lianas, que apresentam como característica reprodutiva a floração e frutificação abundantes e ampla dispersão principalmente por meio do vento, mas podendo ocorrer também por meio da água ou animais (HOLMES, 1995). A morfoespécie de Mikania encontrada não pode ser identificada em seu nível específico, isso ocorreu pois Mikania pertence à família Asteraceae,

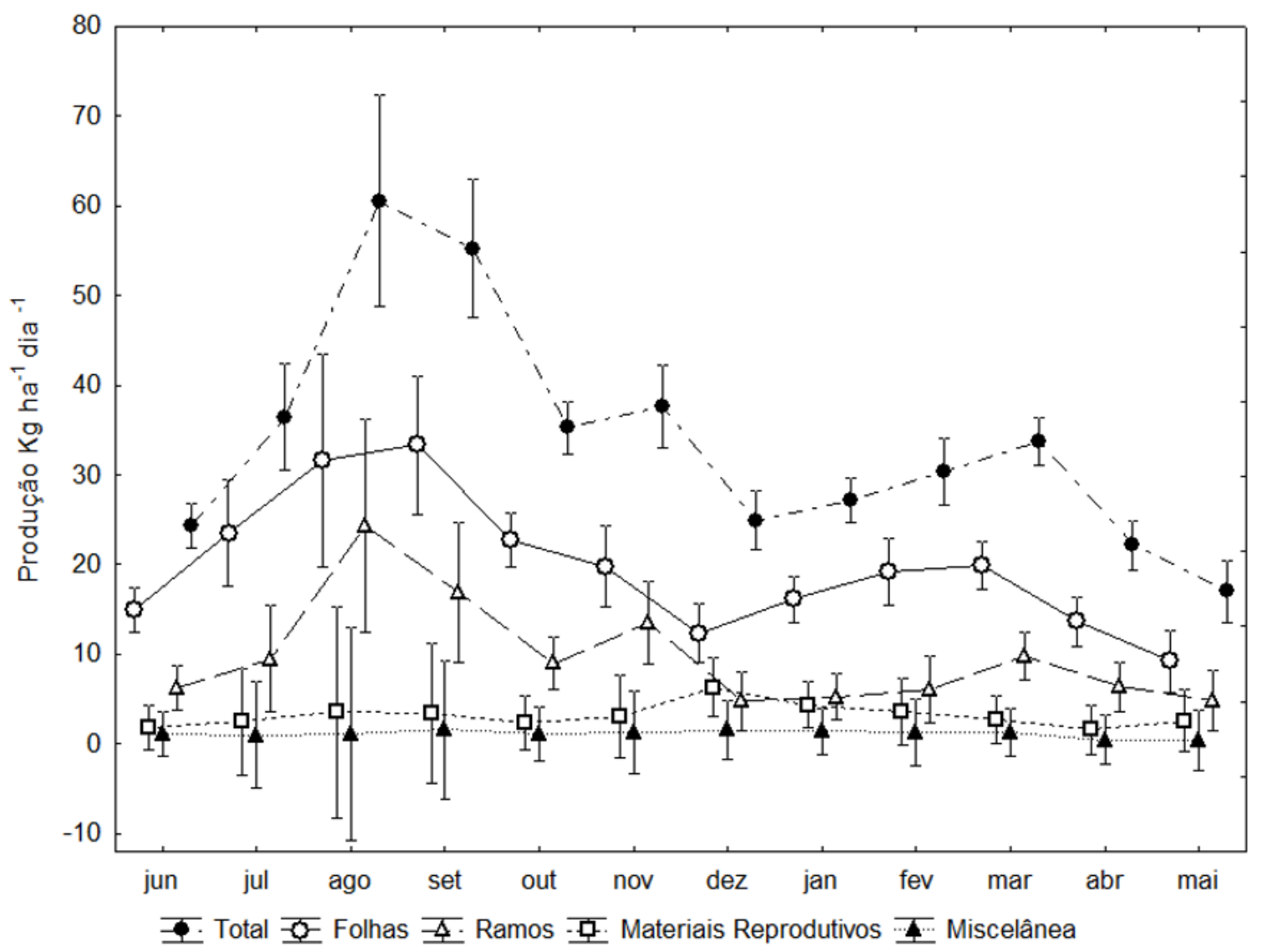

FIGURA 2: Gráfico da produção de serapilheira nas diferentes frações e total $\mathrm{kg} \mathrm{ha}^{-1} \mathrm{dia}^{-1}$ mês ${ }^{-1}$ (média e intervalo de 95\% de confiança) entre jun/2011 e mai/2012 na RPPN Fazenda Santa Maria - PR.

FIGURE 2: Graphic of the litterfall production in different fractions and total in $\mathrm{kg} \mathrm{ha}^{-1} \mathrm{day}^{-1}$ month $^{-1}$ (mean and coefficient of 95\% of variation), between Jun/2011 and May/2012 in Santa Maria Farm's Private Nature Reserve, PR state. 
TABELA 1: Espécies encontradas na chuva de sementes na RPPN Fazenda Santa Maria - PR. TABLE 1: Species found in the seed rain in Santa Maria Farm's Private Nature Reserve, PR state.

\begin{tabular}{|c|c|c|c|c|c|c|c|}
\hline Espécie & Família & FV & CS & SD & $\mathrm{N}$ & DR & Meses \\
\hline Mikania sp. & Asteraceae & $\mathrm{L}$ & $\mathrm{SC}$ & Ane & 6915 & $37,79 \%$ & ago/mar e mai \\
\hline Cecropia pachystachya Trécul & Urticaceae & A & $\mathrm{P}$ & Zoo & 2720 & $14,86 \%$ & mar \\
\hline Pisonia aculeata $\mathrm{L}$. & Nyctaginaceae & $\mathrm{L}$ & $\mathrm{SC}$ & Zoo & 2270 & $12,40 \%$ & todos \\
\hline Gouania ulmifolia Hook. \& Arn. & Rhamnaceae & $\mathrm{L}$ & $\mathrm{SC}$ & Zoo & 2018 & $11,03 \%$ & jun/fev \\
\hline Dendropanax cuneatum (DC.) Decne. \& Planch. & Araliaceae & A & $\mathrm{Si}$ & Zoo & 1197 & $6,54 \%$ & jun, ago/jan e mai \\
\hline Euterpe edulis Mart. & Arecaceae & A & $\mathrm{C}$ & Zoo & 388 & $2,12 \%$ & exceto out, dez e jan \\
\hline Holocalyx balansae Micheli & Fabaceae & A & $\mathrm{C}$ & Zoo & 375 & $2,05 \%$ & exceto jun e mai \\
\hline Cordia trichotoma (Vell.) Arráb. ex Steud. & Boraginaceae & A & St & Ane & 343 & $1,87 \%$ & exceto dez, jan \\
\hline Ocotea diospyrifolia (Meisn.) Mez & Lauraceae & A & $\mathrm{P}$ & Zoo & 235 & $1,28 \%$ & jun, jan/abr \\
\hline Alchornea triplinervia (Spreng.) Müll.Arg. & Euphorbiaceae & $\mathrm{A}$ & St & Zoo & 231 & $1,26 \%$ & ago/fev \\
\hline Serjania sp. & Sapindaceae & $\mathrm{L}$ & $\mathrm{SC}$ & Ane & 194 & $1,06 \%$ & exceto dez, jan e mai \\
\hline Maclura tinctoria (L.) D.Don ex Steud. & Moraceae & A & $\mathrm{Si}$ & Zoo & 141 & $0,77 \%$ & jun, nov/jan, abr e mai \\
\hline Solanum granulosoleprosum Dunal & Solanaceae & A & $\mathrm{P}$ & Zoo & 114 & $0,62 \%$ & jun, abr e mai \\
\hline Ruprechtia laxiflora Meisn. & Polygoniaceae & A & St & Ane & 111 & $0,61 \%$ & jun/nov, abr e mai \\
\hline Syagrus romanzoffiana (Cham.) Glassman & Arecaceae & A & $\mathrm{C}$ & Zoo & 86 & $0,47 \%$ & exceto set e dez \\
\hline Condylocarpon isthmicum (Vell.) A.DC. & Apocynaceae & $\mathrm{L}$ & $\mathrm{SC}$ & Ane & 81 & $0,44 \%$ & todos \\
\hline Senegalia polyphylla (DC.) Britton \& Rose & Fabaceae & $\mathrm{A}$ & $\mathrm{P}$ & Aut & 78 & $0,43 \%$ & jun, ago/nov, fev e mar \\
\hline Balfourodendron riedelianum (Engl.) Engl. & Rutaceae & A & St & Ane & 66 & $0,36 \%$ & jun/set, jan/abr \\
\hline Cedrela fissilis Vell. & Meliaceae & $\mathrm{A}$ & $\mathrm{St}$ & Ane & 63 & $0,34 \%$ & ago/nov, mar e abr \\
\hline Dalbergia frutescens (Vell.) Britton & Fabaceae & $\mathrm{L}$ & $\mathrm{SC}$ & Ane & 63 & $0,34 \%$ & exceto jul e dez \\
\hline Lauraceae sp. 1 & Lauraceae & A & $\mathrm{SC}$ & Zoo & 62 & $0,34 \%$ & set, nov/jan \\
\hline Sorocea bonplandii (Baill.) W.C.Burger et al. & Moraceae & A & $\mathrm{St}$ & Zoo & 53 & $0,29 \%$ & out/jan \\
\hline Cestrum bracteatum Link \& Otto & Solanaceae & A & $\mathrm{P}$ & Zoo & 52 & $0,28 \%$ & jul, dez/mar \\
\hline Fridericia sp. & Bignoniaceae & $\mathrm{L}$ & $\mathrm{SC}$ & Ane & 47 & $0,26 \%$ & ago/dez \\
\hline Hippocratea volubilis L. & Celastraceae & $\mathrm{L}$ & $\mathrm{SC}$ & Ane & 44 & $0,24 \%$ & exceto out, fev e abr \\
\hline Heliocarpus popayanensis Kunth & Malvaceae & $\mathrm{A}$ & $\mathrm{P}$ & Ane & 40 & $0,22 \%$ & set/dez, fev e mar \\
\hline Forsteronia sp. & Apocynaceae & $\mathrm{L}$ & $\mathrm{SC}$ & Ane & 30 & $0,16 \%$ & jun/nov e abr \\
\hline Mascagnia divaricata (Kunth) Nied. & Malpighiaceae & $\mathrm{L}$ & $\mathrm{SC}$ & Ane & 23 & $0,13 \%$ & jun, jan, mar/mai \\
\hline Prunus myrtifolia (L.) Urb. & Rosaceae & A & $\mathrm{Si}$ & Zoo & 22 & $0,12 \%$ & out/jan e abr \\
\hline Casearia sylvestris $\mathrm{Sw}$ & Salicaceae & A & $\mathrm{P}$ & Zoo & 20 & $0,11 \%$ & jun/abr \\
\hline Guarea kunthiana A.Juss. & Meliaceae & A & St & Zoo & 19 & $0,10 \%$ & ago, set e dez \\
\hline Aegiphila integrifolia (Jacq.) Moldenke & Lamiaceae & A & $\mathrm{P}$ & Zoo & 16 & $0,09 \%$ & $\mathrm{fev} / \mathrm{mai}$ \\
\hline Inga marginata willd. & Fabaceae & A & $\mathrm{Si}$ & Zoo & 16 & $0,09 \%$ & out $/$ dez e mar \\
\hline Nectandra megapotamica (Spreng.) Mez & Lauraceae & A & $\mathrm{C}$ & Zoo & 16 & $0,09 \%$ & jun, ago/out, mar e mai \\
\hline Handroanthus ochraceus (Cham.) Mattos & Bignoniaceae & A & $\mathrm{C}$ & Ane & 15 & $0,08 \%$ & nov/jan \\
\hline Dalechampia stipulacea Müll.Arg. & Euphorbiaceae & $\mathrm{L}$ & $\mathrm{SC}$ & Aut & 12 & $0,07 \%$ & jun, nov e fev \\
\hline Poaceae sp. 1 & Poaceae & $\mathrm{H}$ & $\mathrm{SC}$ & Ane & 12 & $0,07 \%$ & jul, set/nov \\
\hline Luehea divaricata Mart. & Malvaceae & A & $\mathrm{Si}$ & Ane & 10 & $0,05 \%$ & $\operatorname{mar}$ \\
\hline Jacaranda micrantha Cham. & Bignoniaceae & A & $\mathrm{P}$ & Ane & 9 & $0,05 \%$ & nov \\
\hline Poaceae sp. 2 & Poaceae & $\mathrm{H}$ & $\mathrm{SC}$ & Ane & 9 & $0,05 \%$ & ago, set e nov \\
\hline Byttneria catalpifolia Jacq. & Malvaceae & $\mathrm{L}$ & $\mathrm{SC}$ & Aut & 5 & $0,03 \%$ & ago e jan \\
\hline Euphorbiaceae sp. 1 & Euphorbiaceae & $\mathrm{SC}$ & $\mathrm{SC}$ & $\mathrm{SC}$ & 5 & $0,03 \%$ & nov/jan e mar \\
\hline Picramnia sp. & Picramiaceae & A & $\mathrm{SC}$ & Zoo & 4 & $0,02 \%$ & $\mathrm{dez} / \mathrm{fev}$ \\
\hline Rauvolfia sellowii Müll.Arg. & Apocynaceae & A & $\mathrm{P}$ & Zoo & 4 & $0,02 \%$ & set, mar e abr \\
\hline Solanum sp. & Solanaceae & A & $\mathrm{SC}$ & Zoo & 4 & $0,02 \%$ & jun e nov \\
\hline Albizia polycephala (Benth.) Killip ex Record & Fabaceae & A & $\mathrm{P}$ & Aut & 3 & $0,02 \%$ & jul e out \\
\hline Fabaceae sp. 1 & Fabaceae & $\mathrm{SC}$ & $\mathrm{SC}$ & $\mathrm{SC}$ & 3 & $0,02 \%$ & mai \\
\hline Ilex paraguariensis A.St.-Hil. & Aquifoliaceae & A & $\mathrm{C}$ & Zoo & 3 & $0,02 \%$ & jun, abr e mai \\
\hline Cordia americana (L.) Gottschling \& J.S.Mill. & Boraginaceae & $\mathrm{A}$ & $\mathrm{P}$ & Ane & 3 & $0,01 \%$ & jul,ago e fev \\
\hline Cordia ecalyculata Vell. & Boraginaceae & A & $\mathrm{P}$ & Zoo & 2 & $0,01 \%$ & jun e abr \\
\hline Lauraceae sp. 2 & Lauraceae & A & $\mathrm{SC}$ & Zoo & 2 & $0,01 \%$ & mar \\
\hline Myrsine umbellata Mart. & Myrsinaceae & A & $\mathrm{Si}$ & Zoo & 2 & $0,01 \%$ & jun \\
\hline Ocotea sp. & Lauraceae & A & $\mathrm{SC}$ & Zoo & 2 & $0,01 \%$ & mar e abr \\
\hline Parapiptadenia rigida (Benth.) Brenan & Fabaceae & A & $\mathrm{Si}$ & Aut & 2 & $0,01 \%$ & set \\
\hline Schefflera calva (Cham.) Frodin \& Fiaschi & Araliaceae & A & $\mathrm{C}$ & Zoo & 2 & $0,01 \%$ & mar \\
\hline Ceiba speciosa (A.St.-Hil.) Ravenna & Malvaceae & A & $\mathrm{Si}$ & Ane & 1 & $0,01 \%$ & mai \\
\hline Chrysophyllum gonocarpum (Mart. \& Eichler ex Miq.) Engl. & Sapotaceae & A & St & Zoo & 1 & $0,01 \%$ & fev \\
\hline Handroanthus impetiginosus Mattos & Bignoniaceae & A & $\mathrm{C}$ & Ane & 1 & $0,01 \%$ & set \\
\hline Koelreuteria paniculata L. & Sapindacea & A & $\mathrm{SC}$ & Ane & 1 & $0,01 \%$ & out \\
\hline Lasiacis ligulata Hitchc. \& Chase & Poaceae & $\mathrm{H}$ & $\mathrm{P}$ & Ane & 1 & $0,01 \%$ & jun \\
\hline Pirostegia sp. & Bignoniaceae & $\mathrm{L}$ & $\mathrm{SC}$ & Ane & 1 & $0,01 \%$ & set \\
\hline Psychotria carthagenensis Jacq. & Rubiaceae & $\mathrm{A}$ & St & Zoo & 1 & $0,01 \%$ & jun \\
\hline Styrax leprosus Hook. \& Arn. & Styracaceae & $\mathrm{A}$ & $\mathrm{P}$ & Zoo & 1 & $0,01 \%$ & mar \\
\hline 14 morfoespécies indeterminadas & \multirow{2}{*}{\multicolumn{7}{|c|}{$\begin{array}{cc}29 & 0,16 \% \\
18300 \quad 100 \%\end{array}$}} \\
\hline $\begin{array}{rr} & \text { Total } \\
\end{array}$ & & & & & & & \\
\hline
\end{tabular}

Em que: (FV) forma de vida: (A) arbórea, (L) liana e (H) herbácea; (CS) categoria sucessional: (P) pioneira, (Si) secundária inicial, (St) secundária tardia e (C) climácica; (SD) síndrome de dispersão: (Ane) anemocórica, (Aut) autocórica, (Zoo) zoocórica e (SC) sem classificação. (N) Número de sementes e (DR) densidade relativa.

(FV) life form: (A) tree, (L) climbers and (H) herbaceous; (CS) successional category: (P) pioneer, (Si) early secondary, (St) late secondary and (C) climax; (SD) dispersion syndrome: (Ane) anemochory, (Aut) autochory, (Zoo) zoochory and $(\mathrm{SC})$ unrated. $(\mathrm{N})$ Number of seeds and (DR) relative density. 
TABELA 2: Trabalhos sobre chuvas de sementes comparados com a RPPN Fazenda Santa Maria - PR.

TABLE 2: Articles about seed rain compared with the Santa Maria Farm's Private Nature Reserve, PR state.

\begin{tabular}{|c|c|c|c|c|c|c|}
\hline Autores & Tipos Vegetacionais & Local & $\begin{array}{l}\text { Tamanho da } \\
\text { Área }\end{array}$ & $\begin{array}{c}\text { Área } \\
\text { Amostrada }\end{array}$ & Espécies & Densidade Absoluta \\
\hline Presente trabalho & $\begin{array}{l}\text { Floresta Estacional } \\
\text { Semidecidual }\end{array}$ & $\begin{array}{c}\text { RPPN Fazenda Santa } \\
\text { Maria - PR }\end{array}$ & 242 ha & $9 \mathrm{~m}^{2}$ & 79 & $\begin{array}{c}2.033,33 \text { sementes } \\
\mathrm{m}^{-2}\end{array}$ \\
\hline $\begin{array}{c}\text { PENHALBER e } \\
\text { MANTOVANI (1997) }\end{array}$ & $\begin{array}{l}\text { Florestas Pluviais na } \\
\text { encosta Atlântica e } \\
\text { Floresta Estacional }\end{array}$ & $\begin{array}{c}\text { Parque Estadual das } \\
\text { Fontes do Ipiranga - SP }\end{array}$ & 345 ha & $10 \mathrm{~m}^{2}$ & 54 & $\begin{array}{c}1.804,4 \text { sementes } \\
\mathrm{m}^{-2}\end{array}$ \\
\hline $\begin{array}{c}\text { GROMBONE- } \\
\text { GUARATINI e } \\
\text { RODRIGUES (2002) }\end{array}$ & $\begin{array}{l}\text { Floresta Estacional } \\
\text { Semidecidual }\end{array}$ & $\begin{array}{l}\text { Reserva Municipal de } \\
\text { Santa Genebra - SP }\end{array}$ & 251,8 ha & $8,75 \mathrm{~m}^{2}$ & 54 & 442 sementes $\mathrm{m}^{-2}$ \\
\hline ARAUJO et al. (2004) & $\begin{array}{l}\text { Floresta Estacional } \\
\text { Decidual Ripária }\end{array}$ & $\begin{array}{l}\text { Fragmento florestal de } \\
\text { Cachoeira do Sul - RS }\end{array}$ & 4 ha & $30 \mathrm{~m}^{2}$ & 50 & $\begin{array}{l}1^{\circ} \text { Ano: } 155 \\
\text { sementes } \mathrm{m}^{-2} \\
2^{\circ} \text { Ano: } 71 \\
\text { sementes } \mathrm{m}^{-2}\end{array}$ \\
\hline CAMPOS et al. (2009) & $\begin{array}{l}\text { Floresta Estacional } \\
\text { Semidecidual }\end{array}$ & $\begin{array}{c}\text { Fragmento florestal de } \\
\text { Viçosa - MG }\end{array}$ & 75 ha & $6,25 \mathrm{~m}^{2}$ & 43 & $\begin{array}{c}1^{\circ} \text { ano: } 113,92 \\
\text { sementes } \mathrm{m}^{-2} \\
2^{\circ} \text { ano: } 2.603,84 \\
\text { sementes } \mathrm{m}^{-2}\end{array}$ \\
\hline
\end{tabular}

que é a segunda família botânica mais diversa do Brasil, com aproximadamente 2.000 espécies (GIULIETTI et al., 2009), além disso, seus diásporos do tipo de aquênio não são caracteres diagnósticos para o reconhecimento de suas espécies.

A elevada produção de Cecropia pachystachya se deu por representar uma espécie de características sucessionais de pioneira. Espécies pioneiras geralmente produzem sementes numerosas e pequenas, apresentam alta capacidade de se depositarem e de permanecerem durante longos períodos de tempo no banco de sementes do solo, além disso, os frutos da espécie são muito utilizados pela fauna, como quirópteros, primatas e aves, sendo, portanto, importante na contribuição da regeneração natural de clareiras e na manutenção e dinâmica das florestas secundárias (CARVALHO, 2006). Tanto a Pisonia aculeata quanto a Gouania ulmifolia são lianas e também foram frequentes na chuva de sementes. As lianas ocorrem de forma abundante em Florestas Estacionais Semideciduais, e por isso seus frutos também são importantes recursos para a fauna, principalmente porque costumam frutificar nos meses em que a frutificação das espécies arbóreas é mais baixa (MORELLATO; LEITÃO-FILHO, 1996). A elevada produção anual e a ampla dispersão de Dendropanax cuneatus são comuns em fragmentos da fisionomia vegetacional estudada (LORENZI et al., 2002).

Pisonia aculeata e Condylocarpon isthmicum, apresentaram maiores frequências de ocorrência ao longo dos meses e apareceram nos 12 meses estudados; Holocalyx balansae, Cordia trichotoma e a Dalbergia frutescens, foram coletadas em oito meses de amostragem; e Mikania sp., Gouania ulmifolia, Euterpe edulis, Serjania sp. e Syagrus romanzoffiana, ocorreram em sete meses (Tabela 1). Entre essas Pisonia aculeata, Holocalyx balansae, Gouania ulmifolia, Euterpe edulis e Syagrus romanzoffiana são espécies zoocóricas, e as altas frequências dessas espécies ao longo do ano estudado representam uma elevada disponibilidade de frutos para a fauna associada.

Em setembro (19\%), outubro (20\%), novembro (27\%) e março $(15 \%)$ foram obtidas as maiores produções de sementes, sendo que setembro, outubro e novembro apresentaram médias significativamente superiores em relação a vários meses analisados $(\mathrm{p}<0,05)$ (Figura 3). Novembro 
apresentou maiores valores devido à elevada produção da Mikania sp., que neste mês representou $74,4 \%$ do total das sementes amostradas. A Cecropia pachystachya também apresentou um pico na produção de sementes no mês de março, com $88,4 \%$ do total de sementes amostradas neste mês, isso ocorreu devido à queda de um ramo com todos os seus frutos e numerosas sementes no coletor.

De acordo com o resultado da Correlação de Spearman $\left(r_{s}\right)$, foi observada a ocorrência de correlação positiva $(r=0,72 ; \mathrm{p}=0,004)$ entre valores mensais médios de serapilheira em $\mathrm{Kg} \mathrm{ha}^{-1}$ dia $^{-1}$ e os de sementes dia $^{-1}$, o que aponta que os períodos de maiores produções de serapilheira estão associados com períodos de maiores produções de sementes.

Em Florestas Estacionais Semideciduais há uma tendência de ocorrerem picos de produções entre setembro e novembro, que este representa o período de atividades vegetativas e reprodutivas mais intensas das espécies. Estes eventos vegetativos e reprodutivos estão relacionados com o fim do período de seca fisiológica (inverno), e também com a alta quantidade de nutrientes presentes no solo, que é causada pela maior deposição de serapilheira nesse período (MORELLATO, 1991; PENHALBER; MANTOVANI, 1997; GROMBONE-GUARATINI; RODRIGUES, 2002; ARAUJO et al., 2004).

A forma de vida predominante foi arbórea com 74,6\%, seguida de lianas com 20,64\% e herbáceas com 4,76\%, resultados semelhantes aos obtidos por Araujo et al. (2004) e Campos et al. (2009), em que foram encontradas predominantemente sementes desta forma de vida, seguidas por espécies de lianas. Como mencionado anteriormente, as Florestas Estacionais Semideciduais apresentam elevada diversidade de lianas, isso ocorre por essa formação apresentar áreas de dossel mais aberto e com maior luminosidade em relação às Florestas Ombrófilas (CARMO; MORELLATO, 2009). Das 47 espécies arbóreas coletadas, apenas 28 foram encontradas no estudo florístico e fitossociológico na área (GRIS; TEMPONI; DAMASCENO JUNIOR, 2014), o que evidencia que estudos sobre a chuva de sementes podem trazer informações complementares aos estudos florísticos e fitossociológicos.

De julho a dezembro mais de $50 \%$ das sementes coletadas foram representadas por lianas, e entre janeiro e maio, as sementes de espécies arbóreas predominaram (Figura 4). Entre julho a setembro, que são meses de baixas temperaturas na região, a elevada produção de sementes de lianas, sugere que estas podem estar contribuindo para manter elevada a disponibilidade de

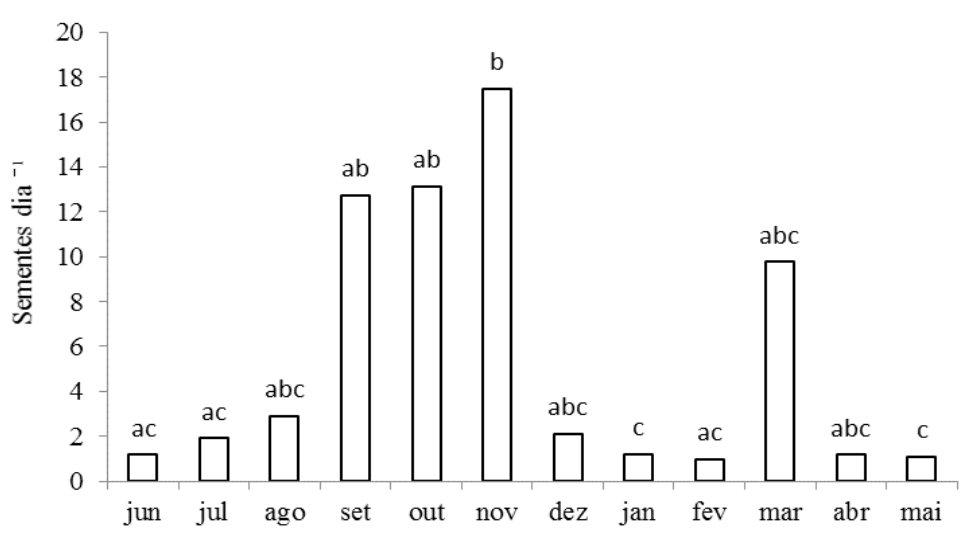

FIGURA 3: Número de sementes dia ${ }^{-1}$ mês $^{-1}$ coletadas entre jun/2011 e mai/2012 na RPPN Fazenda Santa Maria - PR. Letras diferentes indicam diferença entre os meses $(p<0,05)$.

FIGURE 3: Number of seeds day ${ }^{-1}$ month $^{-1}$ collected between Jun/2011 and May/2012 in Santa Maria Farm's Private Nature Reserve, PR state. Different letters indicate difference between the months $(p<0,05)$. 
frutos para a fauna local, uma vez que neste período as sementes de espécies arbóreas são menos representativas. Esse assincronismo entre lianas e arbóreas é característico de Florestas Estacionais secundárias tardias (ENGEL; FONSECA; OLIVEIRA, 1998). Em novembro e dezembro, a elevada frequência de sementes de lianas ocorreu devido à elevada deposição da Mikania sp. A partir de janeiro, a maior deposição de sementes de arbóreas foi semelhante aos resultados obtidos por Penhalber e Mantovani (1997), que encontraram maiores deposições de espécies arbóreas entre dezembro e maio.

Em relação às categorias sucessionais das espécies pertencentes à forma de vida arbórea, $37 \%$ foram representadas por espécies pioneiras, enquanto as secundárias iniciais e tardias apresentaram $22 \%$ cada e as climácicas corresponderam a $20 \%$. Estes dados revelaram uma grande diferença ao serem comparadas com o trabalho de florística e fitossociologia realizado nesta área por Gris, Temponi e Damasceno Junior (2014), que obteve 21\% de espécies pioneira. Essa diferença pode ser explicada por espécies pioneiras terem como característica a produção de um grande número de sementes, desta forma, estas são dispersas e depositadas no banco de sementes do solo e representam uma alta capacidade de regeneração natural em áreas degradadas (BARBOSA et al., 2012). Por estas espécies se reproduzirem rapidamente, são as primeiras a aparecerem na chuva de sementes, mesmo sem serem detectadas no estrato arbóreo adulto (BUDOWSKI, 1965).

A zoocoria predominou entre as síndromes de dispersão ocorrendo em 52,54\% das espécies, enquanto a anemocoria ocorreu em 38,98\% e a autocoria em 8,47\%. Semelhantes aos valores identificados por Carmo e Morellato (2009), que obtiveram 57\% de espécies zoocóricas e 30\% de anemocóricas em Florestas Estacionais Semideciduais. Tais dados corroboram também com os resultados encontrados por Penhalber e Mantovani (1997), sendo 59\% de espécies zoocóricas e $33 \%$ de anemocóricas na chuva de sementes em uma Floresta Estacional Semidecidual.

Ao longo dos meses as sementes zoocóricas foram frequentes, apresentando frequências inferiores as anemocóricas somente nos meses de novembro e dezembro, devido à elevada deposição das sementes de Mikania sp (Figura 5). Estas informações evidenciam a abundante disponibilidade de frutos para a fauna local. Barbosa et al. (2012) ressaltam que a relação planta/ frugívoro na zoocoria é essencial para a conservação de e manutenção dos ecossistemas, além de contribuir para a aceleração de seu processo sucessional, pois os animais têm um papel importante na dispersão das espécies, bem como a adição de várias espécies a uma área (SILVA, 2008).

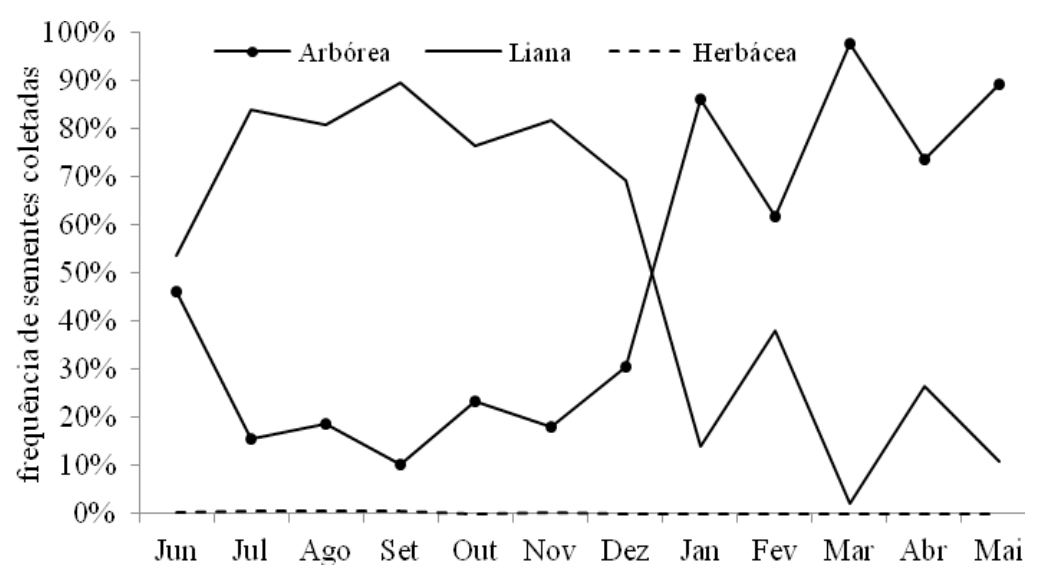

FIGURA 4: Frequência de sementes de espécies arbórea, liana e herbácea, entre jun/2011 e mai/2012, na RPPN Fazenda Santa Maria - PR.

FIGURE 4: Frequency of seeds of trees, climbers and herbaceous species, between Jun/2011 and May/2012, in Santa Maria Farm's Private Nature Reserve, PR state. 


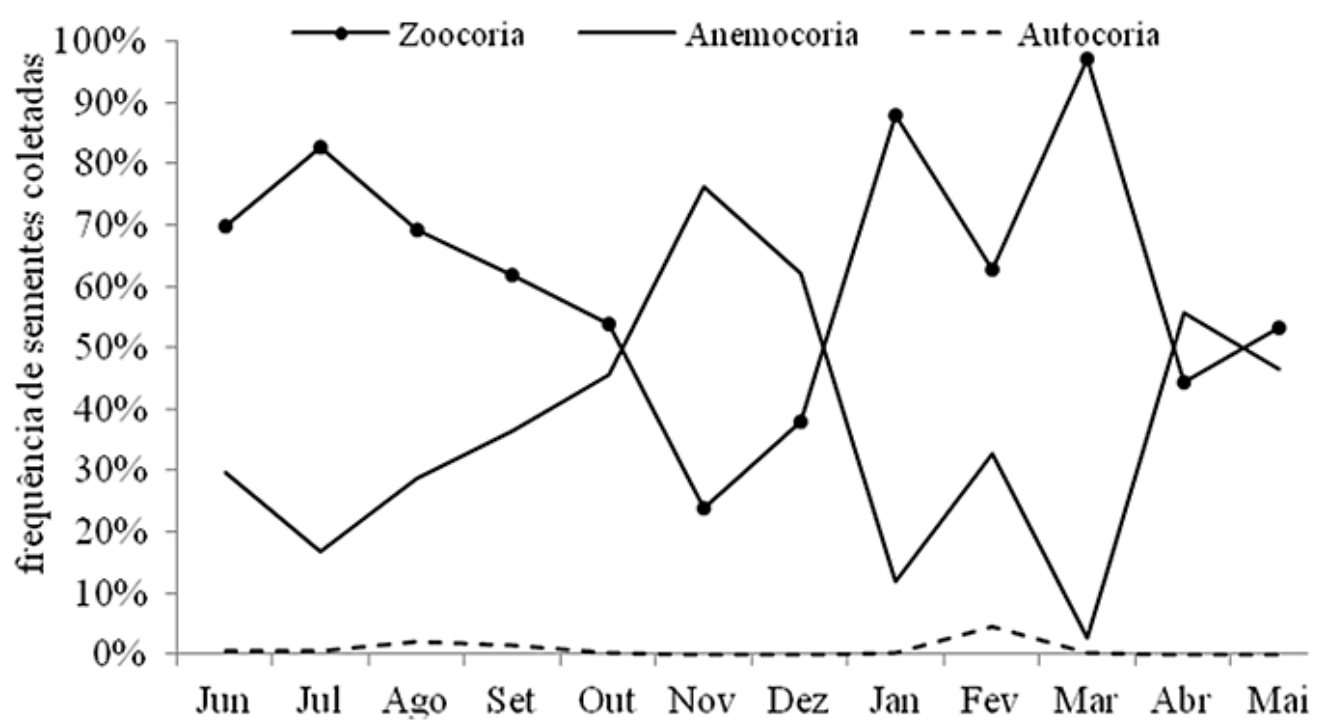

FIGURA 5: Frequência de sementes de espécies zoocóricas, anemocóricas e autocóricas, entre jun/2011 e mai/2012, na RPPN Fazenda Santa Maria - PR.

FIGURE 5: Seed frequencies of zoochoric, anemochoric and autochoric species, between Jun/2011 and May/2012, in Santa Maria Farm's Private Nature Reserve, PR state.

\section{CONCLUSÕES}

A produção de serapilheira da RPPN Fazenda Santa Maria é mais elevada nos meses de agosto e setembro marcando o fim do inverno na região, indicando que a dinâmica da serapilheira na área se comporta como nas florestas nativas tardias de mesma formação florestal.

A chuva de sementes apresenta elevada riqueza de espécies, com aumento na deposição de sementes entre os meses de setembro a novembro, presença do assincronismo entre a produção de frutos por espécies de lianas e arbóreas e predomínio de espécies zoocóricas. Indicando uma elevada disponibilidade de recursos para a fauna durante todo o ano e evidenciando assim a manutenção das interações ecológicas entre plantas e frugívoros, fazendo com que a área apresente um elevado potencial de regeneração natural, pois essas relações são essenciais para a conservação de uma floresta.

\section{AGRADECIMENTOS}

Agradecemos aos administradores da Fazenda Santa Maria por permitirem que a pesquise fosse realizada na RPPN Fazenda Santa Maria, à Fundação PTI C\&T pela concessão de bolsa de mestrado à primeira autora e aos funcionários do Museu Botânico de Curitiba e demais botânicos consultados por auxiliar na identificação das sementes.

\section{REFERÊNCIAS}

ARAUJO, M. M. et al. Caracterização da chuva de sementes, banco de sementes do solo e banco de plântulas em Floreta Estacional Decidual ripária Cachoeira do Sul, RS, Brasil. Scientia Forestalis, 
Piracicaba, n. 66, p. 128-141, 2004.

ARAUJO, R. S. Chuva de sementes e deposição de serrapilheira em três sistemas de revegetação de áreas degradadas na Reserva Biológica de Poço das Antas, Silva Jardim, RJ. 2002. 92 f. Dissertação (Mestrado em Ciências Ambientais e Florestais) - Universidade Federal do Rio de Janeiro, Seropédica, 2002.

BUDOWSKI, G. Distribution of tropical American rain forest species in the light of sucessional processes. Turrialba, Costa Rica, v. 15, n. 1, p. 40-42, 1965.

BARBOSA, J. M. et al. Ecologia da Dispersão de Sementes em Florestas Tropicais. In: MARTINS, S. V. Ecologia de Florestas Tropicais do Brasil. 2. ed. Viçosa: Editora UFV, 2012. p. 85-106.

CAMPOS, E. P. et al. Chuva de sementes em Floresta Estacional Semidecidual em Viçosa, MG, Brasil. Acta Botanica Brasilica, Belo Horizonte, v. 23, n. 2, p. 451-458, 2009.

CARMO, M. R. B.; MORELLATO, L. P. C. Fenologia de árvores e arbustos das matas ciliares da Bacia do Rio Tibagi, estado do Paraná, Brasil. In: RODRIGUES, R. R; FILHO, H. F. L. Matas Ciliares: conservação e recuperação. 2. ed. São Paulo: Fapesp, 2009. p. 125-141.

CARVAlHO, P. E. R. Espécies Arbóreas Brasileiras. Colombo: Embrapa Florestas, 2006. v. 2 , $627 \mathrm{p}$.

ENGEL, V. L.; FONSECA, R. C. B.; OLIVEIRA, R. E. Ecologia de lianas e o manejo de fragmentos florestais. Série Técnica IPEF, Piracicaba, v. 12, n. 32, p. 43-64, 1998.

EWEL, J. J. Litter fall and leaf decomposition in a tropical forest succession in eastern Guatemala. Journal of Ecology, London, v. 64, n. 1, p. 293-308, 1976.

FICAGNA, A. C. Reservas Particulares do Patrimônio Natural Sustentabilidade pelo Turismo. Revista Itinerarium, Rio de Janeiro, v. 2, p. 1-21, 2009.

GIUlietTI, A. M. et al. Plantas Raras do Brasil. Belo Horizonte: Conservação Internacional, 2009. $496 \mathrm{p}$.

GRIS, D.; TEMPONI, L. G.; DAMASCENO JUNIOR, G. A. Structure and floristic diversity of remnant semideciduous forest under varying levels of disturbance. Acta Botanica Brasilica, Belo Horizonte, v. 28, n. 4, p. 569-576, 2014.

GROMBONE-GUARATINI, M. T.; RODRIGUES, R. R. Seed bank and seed rain in a seasonal semi-deciduous forest in south-eastern Brazil. Journal of Tropical Ecology, Cambridge, v. 18, n. 5, p. 759-774, 2002.

HOLMES, W. C. A review preparatory to an infregeneric classification of Mikania (tribe: Eupatorieae). In: HIND, D. J. N.; JEFFREY, C.; POPE, G. V. (Eds.). Advances in Compositae Systematics. Kew: Royal Botanical Gardens, 1995. p. 239-254.

HOWE, H. F.; SMALLWOOD, J. Ecology of seed dispersal. Annual Review of Ecology and Systematics, Palo Alto, v. 13, p. 201-228, 1982.

INSTITUTO AGRONÔMICO DO PARANÁ. Cartas Climáticas do Paraná. 2012. Disponível em: $<$ http://www.iapar.br/modules/conteudo/conteudo.php? conteudo $=863>$. Acesso em: 23 jul. 2012.

IBGE. Manuais técnicos em geociências, manual técnico da vegetação brasileira. Rio de Janeir0: IBGE, 2012.

LOISELLE, B.A.; RIBBENS, E.; VARGAS, O. Spatial and temporal variation of seed rain in a Tropical Lowland Wet Forest. Biotropica, Malden, v. 28, n. 1, p. 82-95, 1996.

LORENZI, H. Árvores brasileiras: manual de identicação e cultivo de plantas arbóreas do Brasil. 4. ed. Nova Odessa: Instituto Plantarum, 2002. v. 1, 384 p.

MACHADO, M. R.; PIÑA-RODRIGUES, F. C. M.; PEREIRA, M. G. Produção de serapilheira como bioindicador de recuperação em plantio adensado de revegetação. Revista Árvore, Viçosa, MG, v. 32, n. 1, p. 143-151, 2008. 
MARTINS, S. V. Recuperação de matas ciliares. Viçosa: Aprenda Fácil, 2001.

MARTINS, S. V.; MIRANDA NETO, A.; RIBEIRO, T. M. Uma abordagem sobre diversidade e técnicas de restauração ecológica. In: MARTINS, S. V. Restauração ecológica de ecossistemas degradados. 1. ed. Viçosa: Editora UFV, 2012. p. 17- 40.

MARTINS, S. V.; RODRIGUES, R. R. Produção de serapilheira em clareiras de uma floresta estacional semidecidual no município de Campinas, SP. Revista Brasileira de Botânica, São Paulo, v. 22, n. 3, p. 405-412, 1999.

MORELLATO, L. P. C. Estudo da fenologia de árvores, arbustos e lianas de uma Floresta Semidecídua no sudeste do Brasil. 176 f. Tese (Doutorado em Biologia) - Universidade Estadual de Campinas, Campinas, 1991.

MORELLATO, L. P. C.; LEITÃO-FILHO, H. F. Reproductive phenology of climbers in a southeastern Brazilian forest. Biotropica, Malden, v. 28, n. 2, p. 180-191, 1996.

PAGANO, S. N.; DURIGAN, G. Aspectos da ciclagem de nutrientes em matas ciliares do oeste do Estado de São Paulo, Brasil. In: RODRIGUES, R. R; LEITÃO FILHO, H. F. Matas Ciliares: conservação e recuperação. 2. ed. São Paulo: Fapesp, 2009. p. 109-123.

PENHALBER, E. F.; MANTOVANI, W. Floração e chuva de sementes em mata secundária em São Paulo, SP. Revista Brasileira de Botânica, São Paulo, v. 20, n. 2, p. 205-220, 1997.

PEZZATTO, A. W.; WISNIEWSKI, C. Produção de serapilheira em diferentes seres sucessionais da Floresta Estacional Semidecidual no Oeste do Paraná. Floresta, Curitiba, v. 36, n. 1, p.111-120, 2006.

PIMENTA, J. A. et al. Produção de serapilheira e ciclagem de nutrientes de um reflorestamento e de uma Floresta Estacional Semidecidual no sul do Brasil. Acta Botanica Brasilica, Belo Horizonte, v. 25, n. 1, p. 53-57, 2011.

PRIMACK, R. B.; RODRIGUES, E. Biologia da conservação. 1. ed. Londrina: Planta, 2001. $328 \mathrm{p}$.

RODRIGUES, R. R.; GANDOLFI, S. Restauração de florestas tropicais: subsídios para uma definição metodológica e indicadores de avaliação e monitoramento. In: DIAS, L. E.; MELLO, J. W. V. (Eds.). Recuperação de áreas degradadas. Viçosa: UFV, 1998. p. 203-215.

RYLANDS, A. B.; BRANDON, K. Unidades de conservação brasileiras. Megadiversidade, Belo Horizonte, v. 1, n. 1, p. 27-35, 2005.

SCARIOT, A. et al. Vegetação e Flora. In: RAMBALDI, D. M.; OLIVEIRA, D. A. S. Fragmentação de ecossistemas: causas, efeitos sobre a biodiversidade e recomendações de políticas públicas. 2 . ed. Brasília: MMA/SBF, 2005. 510 p.

SILVA, W. R. A importância das interações planta-animal nos processos de restauração. In: KAGEYAMA, P. Y. et al. Restauração ecológica de ecossistemas naturais. 1. ed. Botucatu: Fepaf, 2008. p. 77-90.

VAN DER PIJL, L. Principles of dispersal in higher plants. 3. ed. New York: Springer Verlag, 1982. 214 p.

VITAL, A. R. T. et al. Produção de serapilheira e ciclagem de nutrientes de uma Floresta Estacional Semidecidual em zona ripária. Revista Árvore, Viçosa, MG, v. 28, n. 6, p. 793-800, 2004. 